\title{
RE: RADIOLOGICAL CLASSIFICATION OF RENAL ANGIOMYOLIPOMAS BASED ON 127 TUMORS
}

\author{
A. PRANDO
}

Int Braz J Urol. 2003; 29: 208-16

\section{Dear Editor,}

We read the article by Dr. Prando with interest (1). He evaluated the radiological findings of 127 angiomyolipomas (AML) and defined 4 categories depending on the fat content of the tumors. The author defined pattern-I as small $(<1.5 \mathrm{~cm})$, intrarenal, homogenous and predominantly fatty lesions and proposed that these lesions tend to become more heterogenous (with increasing amounts of non-fatty soft tissue, intratumoral vessels or hematoma) if they grow. However, we would like to report a case of a large ruptured renal AML with very high amounts of fatty tissue. She was a 46-year-old woman presented to emergency service because of a sudden occurence of left upper abdominal pain. A plain abdominal film was negative and abdominal ultrasonography showed highly echogenic lesion in the lower pole of left kidney surrounded by a hypoechogenic mass. Enhanced computed tomography (CT) of the abdomen con-

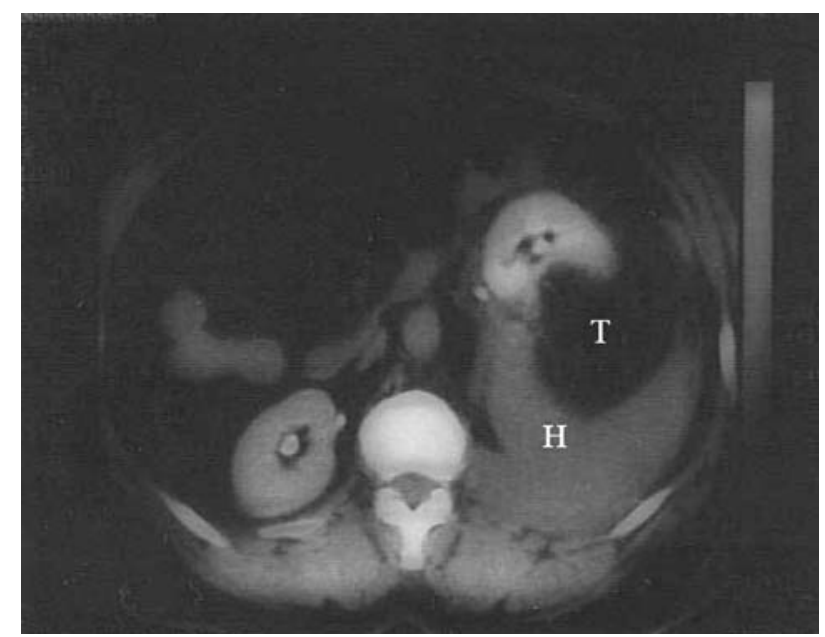

Figure 1 - Left renal AML with perinephric hemorrhage $(H)$. Enhanced CT scan shows homogenous mass with dominant fat attenuation $(T)$. firmed the presence of large perinephric collection with density of blood $(+34 \mathrm{HU})$ including a $9-\mathrm{cm}$ tumoral mass (-85 HU in all regions of the mass) in the center of the hematoma (Figure-1). The patient's general condition deteriorated in a few hours with fall in hemoglobin levels. An emergency operation was planned and nephrectomy with evacuation of a large amount of blood clots was carried out. Pathological examination of the mass revealed AML which had been macroscopically seen as yellow colored fatty tissue in cross-sections. The tumor was demonstrating the histological features belonging to pattern-I in Dr. Prando's categorization system. However, its size was discordant with the same group.

According to our experience, the prevalence of huge AML's with very high amount of fat is very rare. We think that this kind of categorization systems would help identifying imaging characteristics of AML, their differential diagnosis and further therapeutic alternatives for most cases as Dr. Prando had already emphasized in the paper. But, larger series of study groups would provide information of unique categorization systems accepted worldwide.

We thank Dr. Prando for this interesting and insightful article.

Respectfully,

\section{Dr. Ozlem Turksoy \\ Dr. Husnu Tokgoz \\ Dr. Sema Toparli}

Ankara Numune Training and Research Hospital, Department of Radiology (OT, ST) and Ankara Cankaya Hospital, Department of Urology (HT) Ankara, Turkey 